\title{
Perinatal lethal skeletal dysplasia: a case report
}

\author{
Sunita Dubey*, Poonam Goel, Meesha Verma
}

Department of Obstetrics \& Gynaecology, GMCH 32, Sec 32, Chandigarh, India

Received: 05 October 2015

Revised: 24 November 2015

Accepted: 12 December 2015

\section{*Correspondence:}

Dr. Sunita Dubey,

E-mail: sunitas504@gmail.com

Copyright: () the author(s), publisher and licensee Medip Academy. This is an open-access article distributed under the terms of the Creative Commons Attribution Non-Commercial License, which permits unrestricted non-commercial use, distribution, and reproduction in any medium, provided the original work is properly cited.

\section{ABSTRACT}

The word dysplasia originates from ancient Greek words dys (anomalous) and plasia (formation). Skeltal dysplasia (SD) is a heterogeneous group of congenital anomalies characterized by abnormalities in the development of the bone and cartilage tissue. This results in mark disproportion of the long bones, the spine and fetal head relation to the trunk. Perinatal lethal skeletal dysplasia leads to still birth or early neonatal death due to pulmonary hypoplasia. 30 yrs old G3P3L2 at 32 weeks presented with leaking per vaginum. Her serial scan was done as she had previous stillborn male child with short limbs. Her antenatal scan revealed short limbs from 24 weeks. From 18 weeks to 24 weeks she did not underwent any sonography. She went into spontaneous labor and delivered still born male baby with clinical and radiological features suggestive of skeletal dysplasia. Skeletal dysplasia can be diagnosed on antenatal 2 D ultrasound from 14 - 16 weeks onwards. Prenatal genetic testing should be done to diagnose the genetic anomaly and patient should be referred to higher institute for this test. Even if genetic test not done even then termination of pregnancy should be considered based on ultrasound diagnosis especially with family history because of poor fetal prognosis and long term morbidity if survived.

Keywords: Skeletal dysplasia, Osteogenesis imperfect, Perinatal hypophosphatasia, Achondrogenesis, Campomelic dysplasia

\section{INTRODUCTION}

Although each type of skeletal dysplasia is rare, the overall birth prevalence of skeletal dysplasias is estimated to be 2.4 per 10,000 births. ${ }^{1}$ The prevalence during pregnancy appears to be slightly higher at 7.5 per 10,000 ultrasound screened pregnancies. ${ }^{2}$

Skeletal dysplasias may present as either in the form of isolated findings or a phenotypic manifestation of a chromosomal aberration or a genetic disorder. Prenatal diagnosis is mainly on the ultrasound appearance, which is usually achieved during second trimester of pregnancy. Two dimensional ultrasonography may detect the majority of SD, however, difficulty in diagnosis as well as the differential diagnosis are frequently arising. In such cases, further evaluation is needed by the use of additional imaging modalities or by invasive procedures, in order to detect an underlying chromosomal abnormality or a single gene disorder. Our case presented as skeletal dysplasia antenatally and baby was stillborn. Initially, the clinical features were thought to be consistent with the severe lethal form of osteogenesis imperfect (OI) type IIA but radiographic findings seem to be resembled with perinatal lethal hypophosphatasia. We have tried to make a final diagnosis on clinical and radiological finding.

\section{CASE REPORT}

30 yrs old G3P3L2 at 32 week gestation referred with complaints of leaking per vaginum from 1 day. She was 
admitted in antenatal ward for further evaluation. Her ultrasound at 18 weeks revealed single live fetus of 18 weeks with no gross malformation. Her blood pressure and blood sugar were normal. Only routine drugs were used during pregnancy. Her 2 girl child was normal but her last male baby had short limbs who was died after few minutes of birth. There was no history of skeletal dysplasia in the family. In view of previous baby suggestive of osteogenesis imperfect she underwent serial ultrasound.

Repeat ultrasound at 24 weeks was suggestive of single live fetus of 20 weeks with bilateral short femur and humerus. $(\mathrm{BPD} \approx 22$ weeks, $\mathrm{FL} \approx 17$ weeks, $\mathrm{HL} \approx 16$ weeks, $\mathrm{AC} \approx 21$ weeks). She was advised for follow up scan. Repeat scan at 28 weeks revealed single live fetus of 21 weeks small for gestational age with bilateral short femur and humerus length with bowing and under mineralization of skull bones. (BPD $\approx 25$ Weeks, $\mathrm{FL} \approx 19$ Weeks HL $\approx 18$ Weeks $A C \approx 24$ Weeks) All the long bones were found to be less than 2 standard deviations below the mean for gestational age. The remainder of the fetal anatomy was unremarkable and there was adequate amniotic fluid.

On admission she had frank leaking. Ultrasound showed live fetus with breech presentation, absent amniotic fluid with crowding of fetal parts with limited movements of fetal limbs. Neonatal counseling was done. She was explained regarding poor fetal prognosis. We have planned her for termination of pregnancy in view of leaking with poor fetal prognosis. She went in spontanous labour and delivered $800 \mathrm{gm}$ fresh still born male fetus. Skull was thinned and soft. Face was triangular, with protruding open eyes with grossly blue sclera, micrognathia, beaked nose and small humerus and femur s/o micromelia. The chest appeared narrowed and the abdomen was protuberant. Length of the trunk was normal. Skin was dimpled and joints were lax and appear dislocated. In our patient on radiological examination there was diffuse hypomineralizaion of skull and all bones, Wormian bones were absent in skull, ribs were thin, tubular, there was no fracture of any ribs, vertebre and long bones. Some bones like scapula cervical vertebrae, bone of distal part of limbs were not seen. Parents were counseled for autopsy of the baby but denied. DNA testing was not available in our hospital. They were advised to undergo 2D or 3D ultrasound before 20 weeks in next pregnancy. They were also advised to go at higher institute and need of pre-natal genetic testing in future pregnancy. Parents had given consent for photograph and publication of the case.

\section{DISCUSSION}

With the introduction of ultrasound within the routine obstetrical practice, the diagnosis of skeletal anomalies may be possible antanatally. However, despite the progress in imaging techniques and genetics, the correct prenatal diagnosis remain a challenge ${ }^{1}$.This is mainly due to rarity of the disease, the high number of different SD, the phenotype variability of each syndrome, the overlapping features and imaging variation that is related to the gestational age during the examination. Prenatal diagnosis of SD requires a detailed examination by a fetal medicine specialist. The classification of SDs is based on the clinical, the radiological and finally postmortem examination.

The three most common lethal skeletal dysplasias are thanatophoric dysplasia (TD, 29 percent), ${ }^{3}$ osteogenesis imperfect Type 2 (14 percent), and achondrogenesis (9 percent), ${ }^{4}$ which account for 40 to 60 percent of all lethal skeletal dysplasias. ${ }^{1}$ Perinatal type hypophosphatasia, chondroectodermal dysplasia, campomelic dysplasia, and Jeune asphyxiating thoracic dysplasia are also among the more common lethal skeletal dysplasias.

The overall frequency of perinatal deaths due to skeletal dysplasias is approximately 9 per 1000 births, with 23 percent stillborn and an additional 32 percent who do not survive beyond the first week of life $^{1}$ Overall, skeletal dysplasias account for about 5 percent of genetic disorders identified in the newborn period. The neonatal deaths are mainly due to respiratory failure which is due to lung hypoplasia and cerebral hemorrhage. As clinical features are overlapping among these dysplasias we are discussing osteogenesis imperfecta, campomelic dysplasia or bent-limb dysplasia, perinatal type hypophosphatasia and Achondrogenesis.

The incidence of OI is 1:20,000 and occurs in all races and ethnicity. ${ }^{5}$ Inheritance is generally autosomal dominant but new mutations in the sperm or egg are common and recessive inheritance also occurs ${ }^{6,7}$. In most instances (about 90\%), a mutation in one of the two genes (COL1A1 and COL1A2) that encode the chains of type I collagen, the major protein of bone, accounts for the phenotype.

Ultrasound examination, trans-abdominal (TA) or transvaginal (TV), may reveal features of severe, lethal form of OI (OI type II) as early as 13-14 weeks gestation and OI type III is seldom identified before 18 weeks gestation and confirmation of the diagnosis often requires serial ultrasounds to track evolving findings. In our case till 18 weeks bone growth was normal but at 24 weeks scan there is lag of growth. One prenatal ultrasound finding seen in our case can be found in different types of skeletal dysplasia was prominent falx cerebri in the brain under soft non-ossified calvarium due to diffuse demineraliation of the skull (Figure 5). Calvarium appears large relative to face but there was normal cranial size according to gestational age.

In the majority of skeletal dysplasias, there is severe micromelia, femur length will be below -2SD for gestational age but the foot is relatively spared. The femur: foot length ratio is normally 1.0 throughout the pregnancy, thus a ratio of less than 1 is useful in 
distinguishing skeletal dysplasia from other causes of short femur, such as fetal growth restriction or aneuploidy. ${ }^{8}$ Prof Campbell introduced the diagnosis by the ratio of Femur/foot length if $<0.87\left(<5^{\text {th }}\right.$ percentile) a high level of suspicion of SD is raised. ${ }^{8}$

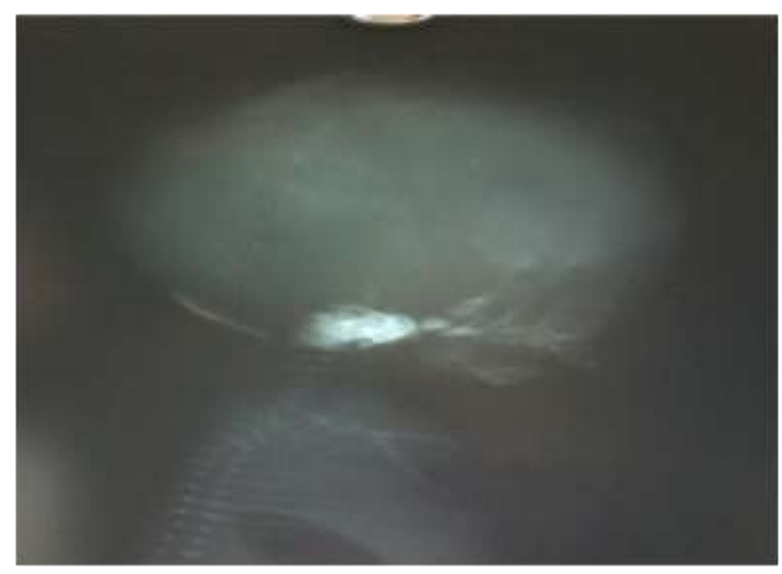

Figure 1: Babygram 1; Diffuse unossified bones of skull and face. Wormian bones in skull were not seen.

Findings most predictive of lethal skeletal dysplasia include ratio of Femur length: abdominal circumference $<0.16,{ }^{9}$ Thoracic circumference $<5$ th percentile, measured at the level of the four-chamber heart view and thoracic:abdominal circumference ratio $<0.6^{9}$ (suggestive of pulmonary hypoplasia associated with the small chest circumference). The earlier in gestation a skeletal dysplasia is detected, the worse the prognosis. The majority of cases found in the first trimester represent lethal skeletal dysplasias.

Increased nuchal translucency during first trimester of pregnancy in fetuses with normal karyotype can be associated with serious SDs, such as achondrogenesis II, achondroplasia, lethal dysplasia, osteogenesis imperfect, partial osteogenesis, osteochondrodysplasia blomstrand, body stalk anomaly and jarcho-Levin syndrome. In most cases, apart from the increased nuchal translucency, the fetus presents with short limbs $\left(<5^{\text {th }}\right.$ percentile $)$ and fetal movements are restricted. The great advantage of $3 \mathrm{D}$ ultrasound is the lower cost, the absence of fetal irradiation and the better understanding of the disease. Diagnosis depends on fetal position and amniotic fluid volume. MRI has a limited role as fetal movements pose many limitations.

OI is characterized by micomelia, hypomineraliation and multiple congenital fractures. Length of the trunk will be short in osteogenesis imperfecta but in our case baby had normal length of the trunk. Platyspondyly and micrognathia are commonly present. These findings can be diagnosed on antenatal ultrasound. ${ }^{10}$ Severe intrauterine growth retardation with pulmonary hypoplasia leads to death in fetal or perinatal period. Those that survive, $80-90 \%$ die by 4 weeks. Clinical features at birth include blue sclera, face has a triangle shape caused by soft craniofacial bones, micrognathia and a beaked nose (Figure 4). Multiple intersutural (wormian) bones along the lambdoid suture could be seen on X-ray of skull. Limbs are angulated and broad due to innumerable fractures and repetitive callus formation. Multiple rib fractures result in a concave thoracic contour, most evident at the lateral thorax where the elbows "bash" in the fragile rib cage.

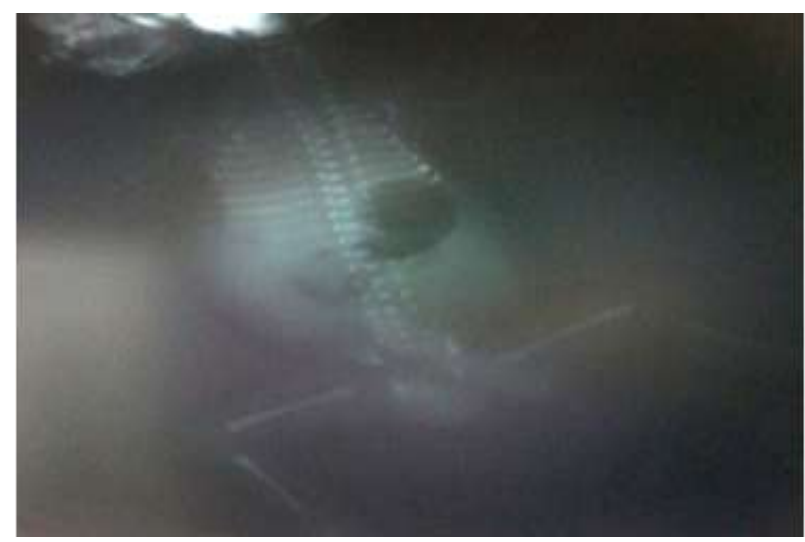

Figure 2: Babygram 2; Long bones \& ribs are thin \& tubular, Cervical vertebrae not visible. Absence of fracture in all bones.

Clinical phenotype (Figure 4) of our case slightly resemble with Pierre Robin Sequence which is characterized by the presence of micrognathia, bird face appearance, glossoptosis and often labio palatine clefting. Pierre Robin Sequence found in campomelic dysplasia or bent-limb dysplasia that is a rare autosomal-dominant condition, usually due to new dominant mutation in the SOX9 gene (sex-determining protein homeobox 9 mapped to $17 \mathrm{q} 24$. Ultrasound features ${ }^{11}$ include macrocephaly, short femur, tibia that are ventrally bowed, cleft palate, club foot and phenotypic reversal of $\operatorname{sex}^{12}$ found in $75 \%$ of cases which were not seen in our case. Other differentiating features are absence of micromelia and presence of normal echogenicity of bones in this sequence. Hypoplastic or absent fibula, hypoplastic scapula and cervical vertebrae (present in 63 percent of cases) may be seen. Other conditions involves congenital heart disease (present in 33 percent of cases), brain and renal abnormalities most cases (77 percent) are lethal because of respiratory insufficiency from laryngotracheomalacia in combination with a mildly narrowed thorax.

On Babygram of our case wormian bones in skull were absent (Figure 1), long bones were thin and straight, ribs were thin, short, gracile, and there were no fractures in any of the bones (Figure 2). There were absences of scapula, small pelvic bones, radiolucent bones of hands and cervical vertebrae (Figure 3). Which compel us to think for other differential diagnosis which includes, and autosomal recessive (severe infantile) hypophosphatasia. achondrogenesis types IA, IB and II, Thanatophoric dysplasia. 


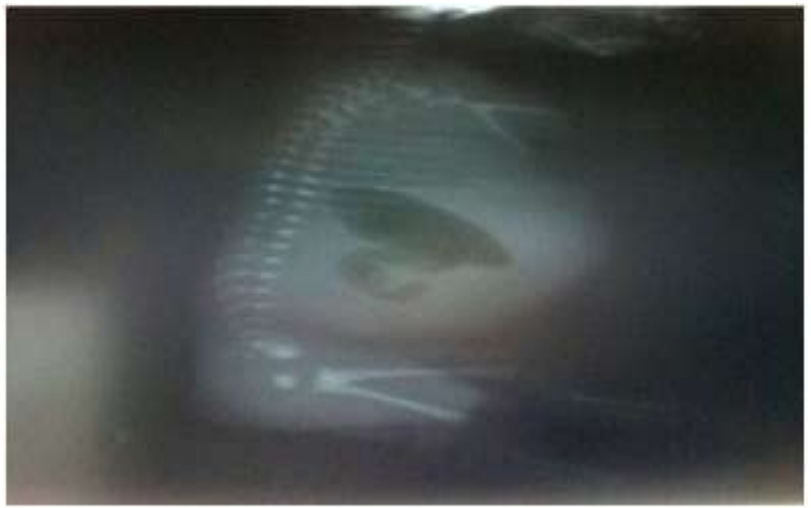

Figure 3: Babygram 3; Small pelvic bones, scapula not visible, bones of arm and hands are radiolucent.

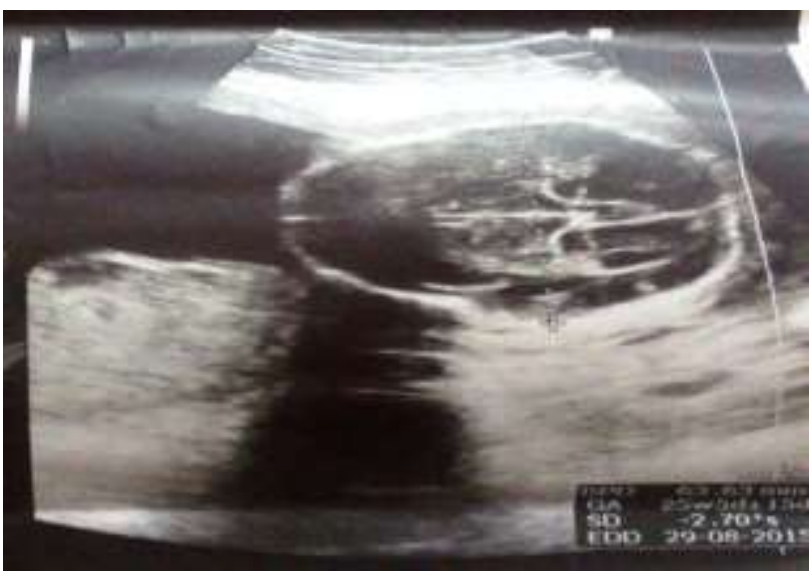

Figure 4: Prenatal ultrasound of the head with lack of shadowing from the unossified skull shows better than normal visualisation of the brain due to unmineralised cranium.

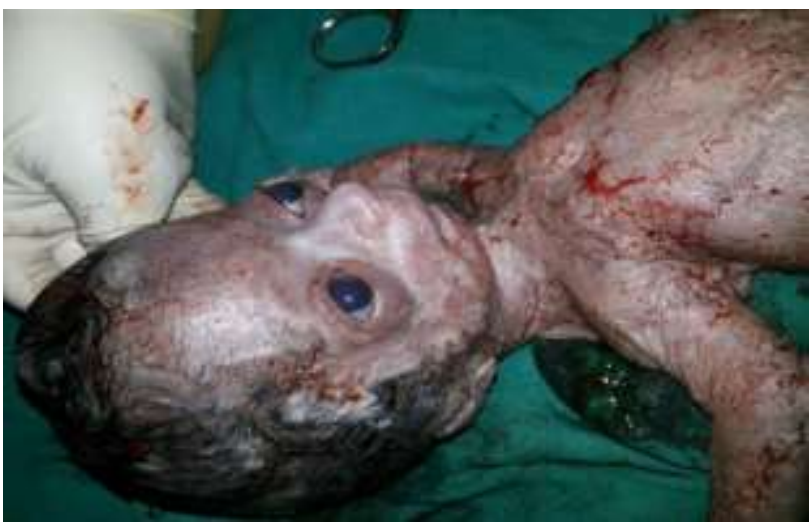

Figure 5: Triangular face, beaked nose, blue sclera and protruding eyes.

Perinatal hypophosphatasia is a genetic disorder (frequency of $1 / 100,000$ ) that is characterised by deficient activity of the enzyme alkaline phosphatase in the bone which results in abnormal unossified bones with variable involvement. Mutations in the $A L P L$ gene (1p36.12) are known to cause hypophosphatasia. Both autosomal- dominant and recessive inheritance has been reported for the mild forms, but only autosomal-recessive inheritance has been implicated in the lethal infantile form. ${ }^{13}$

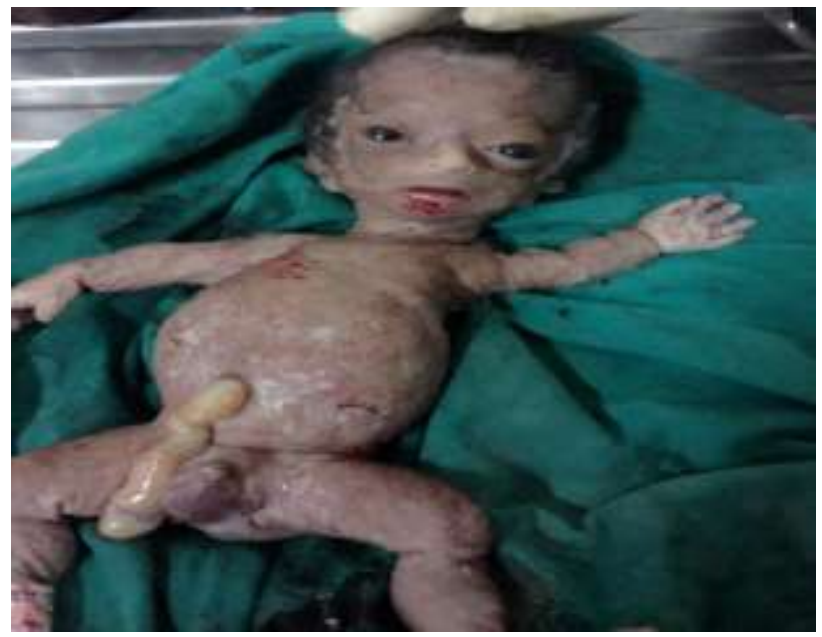

Figure 6: Dimpled skin, small chest with distended abdomen, all joints appears dislocated.

One specific prenatal sonographic area to help differentiate between the two is the infant's hands. The bones of the hands are sonolucent in patients with hypophosphatasia but are echogenic in patients with osteogenesis imperfecta. ${ }^{13}$ Polyhydramnios \& intrautrtine growth retardation may be diagnosed on ultrasound. Infants with perinatal hypophosphatasia may experience pulmonary insufficiency; it is the most frequent cause of death. Hypercalcemia is common and may be associated with apnea or seizures.

Postnatal radiographs will demonstrate a variable appearance of non-ossified bones, osteopenia and some segments of more normal ossification. The bones appears thin, delicate or entirely absent. ${ }^{13}$ Cortical bone could not be made out due to decreased ossification. Some bones like scapula, cervical vertebrae, and bones of hands are also not visualized in our case. In perinatal hypophosphatesia ${ }^{14}$ ossification of ribs and vertebrae is often patchy, as if someone has erased individual ribs or part of spine. In achondrogenesis the undermineralized vertebral bodies become progressively smaller before they disappear completely. In osteogenesis imperfect, mineralization is uniformly reduced and absent vertebrae are uncommon.The skull size is typically normal, ${ }^{15}$ and the cranial vault is compressible under gentle transducer pressure. Extremities are echogenic in osteogenesis imperfeta but sonolucent on ultrasound in hypophosphatasia. Large skin-covered thorn like structures (spurs) which extend from the lateral and medial sides of the knee joints and smaller ones from the lateral side of both elbows are diagnostic of hypophosphatasia. These are osteochondral projections at right angles to the mid-diaphysis of the long bones of the arms and legs which are called Bowder spur. ${ }^{16}$ Our case did not have these spurs however we did finds 
radiolucent bones of the extremities which help in making the diagnosis of hypophosphatasia.

Achondrogenesis is a common lethal skeletal dysplasia, with a prevalence of 0.09 to 0.23 per 10,000 births. Although a phenotypically and genetically diverse group of chondrodysplasias, on ultrasound ${ }^{13,17}$ they generally have severe micromelia, small thoracic circumference.

Macrocrania, short trunk length and decreased mineralization of pubic bone, ischium, most marked in the vertebral bodies giving a "zipper" appearanc are differentiating features from hypophosphatasia. In hypophosphatasia, tubular bones are formed but defective; while in achondrogenesis, in particular type 1, tubular bones are seen as clumps of disorganized bone tissues with arms and legs looked flipper-like.

Thanatophoric dysplasia ${ }^{18}$ (TD) is the most common lethal skeletal dysplasia, with a prevalence of 0.24 to 0.69 per 10,000 births. Severe micromelia with rhizomelic predominance typically, the extremities are so foreshortened that they protrude at right angles to the body is characterictic. Macrocrania and normal mineralization of bone are two other differentiating features from other skeletal dysplasia.

A review of the X-rays findings like thin and tubular long bones and ribs, patchy ossification pattern, absence of scapulae, absence of body of cervical vertebrae and bones of hands, small pelvic bones and absence of fracture suggested that the radiographic findings were more in favour with perinatal lethal hypophosphatasia. Regardless of the time at which the diagnosis is suspected, the first line of evaluation is a detailed medical history, family history, physical examination, appropriate radiographs and routine lab testing. If these do not lead to diagnosis, then specialized genetic testing is warranted.

\section{Prenatal genetic diagnosis}

For most disorders, the gene(s) in which causative mutations lie are known but, because most mutations are unique to a family or individual, their identification may take several weeks. As a result, a discussion about pregnancy continuation or termination generally rests on sonographic evaluation.

The specific diagnosis of skeletal dysplasia including OI, can be determined by biochemical study of cultured cells or analysis of DNA from the fetus. Detailed postmortem evaluation and x-rays of the fetus are a necessary adjunct to diagnosis. If the pregnancy is continued to delivery, the genetic evaluation can be completed in the newborn period. Mutation analysis can be performed for all types of OI, if the mutation in the family is known, using DNA extracted from CVS cells or amniocytes. The time from initial tissue sampling to test result is about 7-14 days.
Prenatal assessment of severe hypophosphatasia may be performed in couples with a previous affected child or a previous affected pregnancy. Mutation analysis of chorionic villus DNAs is now well documented. It seems that mutation analysis is more reliable than AP assay of chorionic villus sampling at least for heterozygote detection where low AP values may be misinterpreted. In pregnancies with clinical symptoms detected by ultrasound but no familial history of hypophosphatasia, the prenatal diagnosis by mutation analysis remains possible. However, such analyze is difficult, due to the time needed for the $A L P L$ gene sequencing, and may not always lead to a result.

According to a recent study (Cubert et al) ${ }^{19}$, cesarean delivery did not decrease fracture rates at birth in infants with nonlethal forms of OI, nor did it prolong survival for those with lethal forms. This study also found that most cesarean deliveries were done for the usual obstetric indications and not specifically because OI was detected in the fetus.

Planning for the delivery should also include conferring with the hospital's neonatologist, chief obstetrical nurse and nursery staff. Medical personnel who have experience with premature infants often have the skills necessary to handle a tiny, fragile baby who have skeletal dysplasias.

\section{CONCLUSIONS}

When fetal skeletal dysplasia is suspected on ultrasound the patient should be referred promptly to a tertiary center for specialized fetal evaluation. The primary role of antenatal diagnosis should be to predict lethality and secondary goals should be to determine the diagnosis or narrow the differential diagnosis. This case clearly demonstrates the importance of performing cytogenetic, radiographic, pathologic and molecular investigations whenever possible, for confirmation of the diagnosis. We were not able to do the genetic analysis of fetus and couples as facility for that is not available at our institute and may not be available everywhere. That's why there is a need to diagnose it by ultrasound so that termination of pregnancy can be done earliest.

Parental consent for publication that included photos and radiographs were kindly given and signed.

Funding: No funding sources

Conflict of interest: None declared

Ethical approval: The study was approved by the Institutional Ethics Committee

\section{REFERENCES}

1. Camera G, Mastroiacovo P. Birth prevalance of skeletal dysplasias in the Italian multicentric monitoring system for birth defects. Prog Clin Biol Res. 1982;104:441. 
2. Weldner BM, Persson PH, Ivarsson SA. Prenatal diagnosis of dwarfism by ultrasound screening. Arch Dis Child. 1985;60:1070.

3. Waller DK, Correa A, Vo TM. The population-based prevalence of achondroplasia and thanatophoric dysplasia in selected regions of the US. Am J Med Genet A. 2008;146A:2385.

4. Connor JM, Connor RA, Sweet EM. Lethal neonatal chondrodysplasias in the West of Scotland 19701983 with a description of a thanatophoric, dysplasialike, autosomal recessive disorder, Glasgow variant. Am J Med Genet. 1985;22:243.

5. Byers PH, Steiner RD. Osteogenesis imperfecta. Annu Rev Med. 1992;43:269-82.

6. Ward LM, Rauch F, Travers R, Chabot G, Azouz EM, Lalic L. Osteogenesis imperfecta type VII: An autosomal recessive form of brittle bone disease. Bone. 2002;31:12-8.

7. Glorieux FH, Rauch F, Plotkin H, Ward L, Travers R, Roughley P. Type V osteogenesis imperfecta: A new form of brittle bone disease. J Bone Miner Res. 2000;15:1650-8.

8. Campbell J, Henderson A, Campbell S. The fetal femur/foot length ratio: A new parameter to assess dysplasia limb reduction, Obstet Gynecol. 1988;72:181-4.

9. Krakow D, Lachman RS, Rimoin DL. Guidelines for the prenatal diagnosis of fetal skeletal dysplasias. Genet Med. 2009;11:127.

10. Munoz C, Filly RA, Golbus MS. Osteogenesis imperfecta type II: prenatal sonographic diagnosis. Radiology. 1990;174:181.

11. Mansour S, Hall CM, Pembrey ME, Young ID. A clinical and genetic study of campomelic dysplasia. J Med Genet. 1995;32:415.
12. Velagaleti GV, Bien-Willner GA, Northup JK. Position effects due to chromosome breakpoints that map approximately $900 \mathrm{~Kb}$ upstream and approximately $1.3 \mathrm{Mb}$ downstream of SOX9 in two patients with campomelic dysplasia. Am J Hum Genet. 2005;76:652.

13. Taybi H, Lachman RS. Radiology of Syndromes, Metabolic Disorders and Skeletal Dysplasias. 4th ed.Philadelphia: Mosby. 1996:628-30.

14. Zankl A, Mornet E, Wong S. Specific ultrasonographic features of perinatal lethal hypophosphatesia. Am J Med Genet Part A. 2008;146A:1200-4.

15. Glanc P, Chitayat D, Unger S. The fetal musculoskeletal system. In: Rumack CM, Wilson SR, Charboneau JW, Levine D, eds. Diagnostic ultrasound. 4th ed. Philadelphia: Elsevier Mosby. 2011:1389-423.

16. Vandevijver N, De Die-Smulders CE, Offermans JP, Van Der Linden ES, Arends JW, Sastrowijoto SH. Lethal hypophosphatasia, spur type: case report and fetopathological study. Genet Couns. 1998;9:205-9.

17. Hall CM. International nosology and classification of constitutional disorders of bone (2001). Am J Med Genet. 2002;113:65.

18. Chen CP, Chern SR, Shih JC. Prenatal diagnosis and genetic analysis of type I and type II thanatophoric dysplasia. Prenat Diagn. 2001;21:89.

19. Rachel C, Cheng EY, Mack S, Pepin MG, Byers PH. Osteogenesis Imperfecta: Mode of Delivery and Neonatal Outcome. Obstetrics \& Gynecology. 2001;97 (1).

Cite this article as: Dubey S, Goel P, Verma M.

Perinatal lethal skeletal dysplasia: a case report. Int J Reprod Contracept Obstet Gynecol 2016;5:224-9. 